\title{
Absolutely Continuous Functions of Several Variables and Quasiconformal Mappings
}

\author{
S. Hencl
}

\begin{abstract}
We prove that functions with bounded $n$-variation and $n$-absolutely continuous functions of $n$-variables in the sense of [4] are stable under quasiconformal mappings. The class of quasiconformal mappings is the best possible since every homeomorphism which induces a bounded operator between $B V^{n}$ spaces is a quasiconformal mapping.
\end{abstract}

Keywords: Absolute continuity in several variables, quasiconformal maps

AMS subject classification: 26B30

\section{Introduction}

Absolutely continuous functions of one variable are admissible transformations for the change of variables in Lebesgue integral. Recently J. Malý [6] introduced a class of $n$-absolutely continuous functions giving an $n$-dimensional analogue of the notion of absolute continuity from this point of view. We study a modified class of $n$-absolutely continuous functions suggested by Zajíček which was introduced in [4]. Our aim is to find the largest class of transformations which preserves $n$-absolute continuity.

Suppose that $\Omega \subset \mathbb{R}^{n}$ is an open set and $0<\lambda<1$. We say that a function $f: \Omega \rightarrow \mathbb{R}^{m}$ is $n, \lambda$-absolutely continuous if for each $\varepsilon>0$ there is a $\delta>0$ such that, for each disjoint finite family $\left\{B_{i}\left(x_{i}, r_{i}\right)\right\}$ of balls in $\Omega$,

$$
\sum_{i} \mathcal{L}_{n}\left(B_{i}\right)<\delta \Longrightarrow \sum_{i}\left(\operatorname{osc}_{B_{i}\left(x_{i}, \lambda r_{i}\right)} f\right)^{n}<\varepsilon
$$

S. Hencl: Dept. Math. \& Stat., P.O. Box 35 (MaD), FIN-40014 Univ. of Jyväskylä; hencl@karlin.mff.cuni.cz

This research has been supported in part by the Research Project MSM 113200007 from the Czech Ministry of Education, Grant No. 201/00/0767 from the Grant Agency of the Czech republic (GA ČR)

ISSN 0232-2064 / \$2.50 C Heldermann Verlag Berlin 
Absolute continuity from [6] coincides with $n, 1$-absolute continuity. It is proved in $[4,6]$ that $n, \lambda$-absolute continuity implies continuity, weak differentiability with gradient in $L^{n}$, differentiability almost everywhere and a formula on change of variables.

It was shown by Csörnyei [1] that there exists a 2,1-absolutely continuous function with respect to balls, which is not a function of this type with respect to cubes, where the concept in question is defined by an obvious modification to the definition given above. On the contrary, $n, \lambda$-absolute continuity does not depend on the shape of the "ball" in the definition for $0<\lambda<1$ (see [4] for details). The class of absolutely continuous functions also does not depend on the precise value of $\lambda$ if $0<\lambda<1$ (see Theorem 3.5 below). From this point of view it is more natural to work with the new definition (i.e. with $0<\lambda<1)$.

Given a measurable set $A \subset \mathbb{R}^{n}$ and a function $f: A \rightarrow \mathbb{R}^{m}$, we define the $n, \lambda$-variation of $f$ on $A$ by

$$
V_{\lambda}^{n}(f, A)=\sup \left\{\sum_{i}\left(\operatorname{osc}_{B\left(x_{i}, \lambda r_{i}\right)} f\right)^{n}: \begin{array}{l}
\left\{B\left(x_{i}, r_{i}\right)\right\} \text { is a disjoint } \\
\text { finite family of balls in } A
\end{array}\right\} .
$$

We denote by $B V_{\lambda}^{n}(\Omega)$ the class of all functions such that $V_{\lambda}^{n}(f, \Omega)<\infty$, define the space $A C_{\lambda}^{n}(\Omega)$ as the family of all $n, \lambda$-absolutely continuous functions in $B V_{\lambda}^{n}(\Omega)$ and write $A C_{\lambda, \text { loc }}^{n}$ for the class of all functions $f$ such that $f \in A C_{\lambda}^{n}(K)$ for every compact set $K \subset \Omega$.

We prove in Section 3 that if $\Omega \subset \mathbb{R}^{n}(n \geq 2)$ is an open set, $0<\lambda<1$ and $F: \Omega \rightarrow \mathbb{R}^{n}$ is a quasiconformal mapping, then

(i) $f \in B V_{\lambda}^{n}(\Omega) \Longleftrightarrow f \circ F^{-1} \in B V_{\lambda}^{n}(F(\Omega))$

(ii) $f \in A C_{\lambda}^{n}(\Omega) \Longleftrightarrow f \circ F^{-1} \in A C_{\lambda}^{n}(F(\Omega))$.

This extends the result from [4] where $F$ was a bi-Lipschitz mapping. Note that the class $A C_{1}^{n}$ is not stable even under bi-Lipschitz mappings (see [5] for details).

Using ideas from [2] we prove the following result in Section 4:

Let $0<\lambda \leq 1$ and $n \geq 2$. If a homeomorphism $F: \Omega \rightarrow \mathbb{R}^{n}$ induces a bounded operator from $B V_{\lambda}^{n}(F(\Omega))$ to $B V_{\lambda}^{n}(\Omega)$, then $F$ is a quasiconformal mapping.

It follows that the results in Section 3 are sharp. 


\section{Preliminaries}

Throughout the paper we consider an open set $\Omega \subset \mathbb{R}^{n}(n>1)$. We denote

- by $\mathcal{L}_{n}(A)$ or $|A|$ the $n$-dimensional Lebesgue measure of a set $A \subset \mathbb{R}^{n}$

- by $\lambda$ a real number $0<\lambda<1$

- by $B(x, r)$ the $n$-dimensional Euclidean open ball with center $x$ and diameter $r$ (throughout the paper we use the letter $B$ for balls only)

- by $\overline{B(x, r)}$ the corresponding closed ball

- $\lambda B=B(x, \lambda r)$ for a given ball $B=B(x, r)$

- by $S(x, r)=\left\{y \in \mathbb{R}^{n}:|x-y|=r\right\}$ a sphere

- by $\operatorname{osc}_{A} f$ the oscillation of $f: \Omega \rightarrow \mathbb{R}^{m}$ over the set $A \subset \Omega$, which is the diameter of $f(A)$

- by $F^{\prime}(x)$ for a mapping $F: \Omega \rightarrow \mathbb{R}^{n}$ the Jacobi matrix of all partial derivatives of $F$ at $x$

- by $\nabla F$ the weak (distributional) derivative

- by $J_{F}(x)$ the determinant of the Jacobi matrix of $F(x)$

- by $W^{1, p}(\Omega)$ and $W_{\text {loc }}^{1, p}(\Omega)$ the Sobolev spaces.

A mapping $F: \Omega \rightarrow \mathbb{R}^{n}$ is called a homeomorphism if there exists its inverse $F^{-1}$ and both $F$ and $F^{-1}$ are continuous. We write $f \circ F$ or $F^{\star} f$ for the composition of the functions $F: \Omega \rightarrow \mathbb{R}^{n}$ and $f: F(\Omega) \rightarrow \mathbb{R}^{m}$; that is $(f \circ$ $F)(x)=\left(F^{\star} f\right)(x)=f(F(x))$ for every $x \in \Omega$. We say that a homeomorphism $F: \Omega \rightarrow \mathbb{R}^{n}$ induces a bounded operator $F^{\star}: B V_{\lambda}^{n}(F(\Omega)) \rightarrow B V_{\lambda}^{n}(\Omega)$ if there is a constant $C>0$ such that $V_{\lambda}^{n}\left(F^{\star} f, \Omega\right) \leq C V_{\lambda}^{n}(f, F(\Omega))$ for every $f \in B V_{\lambda}^{n}(F(\Omega))$.

We use the convention that $C$ denotes a generic positive constant which may change from expression to expression.

\section{Stability of $A C_{\lambda}^{n}$ under quasiconformal mappings}

In this section we will prove that classes of functions $A C_{\lambda}^{n}$ and $B V_{\lambda}^{n}$ are stable with respect to quasiconformal change of variables.

Definition 3.1. Let $1 \leq K<\infty$. A mapping $F: \Omega \rightarrow \mathbb{R}^{n}$ is called $K$-quasiconformal, if it satisfies the following properties:

(i) $F$ is a homeomorphism

(ii) $F \in W_{\text {loc }}^{1, n}\left(\Omega, \mathbb{R}^{n}\right)$

(iii) $|\nabla F(x)|^{n} \leq K\left|J_{F}(x)\right|$ for almost every $x \in \Omega$.

We say that a mapping $F$ is quasiconformal, if there is $K<\infty$ such that $f$ is $K$-quasiconformal.

For the history and basic properties of quasiconformal mappings we refer the reader to [8]. 
Definition 3.2. A function $F: \Omega \rightarrow \mathbb{R}^{n}$ is $\eta$-quasisymmetric if there is a homeomorphism $\eta:[0, \infty) \rightarrow[0, \infty)$ such that, for every $a, b, x \in \Omega$ and $\rho \geq 0$,

$$
|a-x| \leq \rho|b-x| \quad \Longrightarrow \quad|F(a)-F(x)| \leq \eta(\rho)|F(b)-F(x)| .
$$

The following theorem [6: Theorem 2.4] states that quasiconformal mappings are locally quasisymmetric.

Theorem 3.3. Suppose $n \geq 2, F: \Omega \rightarrow \mathbb{R}^{n}$ is a K-quasiconformal mapping and $x_{0} \in \Omega, \alpha>1, r>0$ and $B\left(x_{0}, \alpha r\right) \subset \Omega$. Then $\left.F\right|_{B\left(x_{0}, r\right)}$ is $\eta$-quasisymmetric where $\eta$ depends only on $n, K$ and $\alpha$.

Using this theorem for $\alpha=2$ and a quasiconformal mapping $F: \Omega \rightarrow \mathbb{R}^{n}$, there is $0<\rho_{0}<1$ such that, for a fixed $x \in \Omega$ and $r<\frac{\rho_{0}}{2} \operatorname{dist}(x, \partial \Omega)$,

$$
\sup _{\{a:|x-a| \leq r\}}|F(x)-F(a)| \leq \frac{1}{4} \inf _{\left\{b:|x-b|=\frac{r}{\rho_{0}}\right\}}|F(x)-F(b)| .
$$

Lemma 3.4. Let $0<\lambda \leq 1, f \in B V_{\lambda}^{n}(\Omega)$ and $f \in A C_{\lambda, \text { loc }}^{n}(\Omega)$. Then $f \in A C_{\lambda}^{n}(\Omega)$.

Proof. Fix $\varepsilon>0$. It is not difficult to see from the definition of $n, \lambda$ variation that we can find a finite collection of pairwise disjoint balls $B\left(x_{i}, r_{i}\right)$ such that $\overline{B\left(x_{i}, r_{i}\right)} \subset \Omega$ and

$$
\sum_{i}\left(\operatorname{osc}_{B\left(x_{i}, \lambda r_{i}\right)} f\right)^{n}>V_{\lambda}^{n}(f, \Omega)-\varepsilon
$$

Since $\Omega$ is open and $\overline{B\left(x_{i}, r_{i}\right)} \subset \Omega$, we can find $k \in \mathbb{N}$ such that for

$$
\Omega_{k}=\left\{x \in \Omega:|x|<k \text { and } \operatorname{dist}(x, \partial \Omega)>\frac{1}{k}\right\}
$$

we have $B\left(x_{i}, r_{i}\right) \subset \Omega_{k}$ for each $i$ and therefore $V_{\lambda}^{n}\left(f, \Omega_{k}\right)>V_{\lambda}^{n}(f, \Omega)-\varepsilon$. From this fact and $V_{\lambda}^{n}\left(\Omega_{k}\right)+V_{\lambda}^{n}\left(\Omega \backslash \Omega_{k}\right) \leq V_{\lambda}^{n}(\Omega)$ we obtain $V_{\lambda}^{n}\left(\Omega \backslash \Omega_{k}\right)<\varepsilon$.

For a given $\varepsilon$ we can find $\delta_{1}$ from the definition of $A C_{\lambda}^{n}\left(\Omega_{k+1}\right)$ for $f$. Put

$$
\delta=\min \left\{\delta_{1}, \mathcal{L}_{n}\left(B\left(0, \frac{1}{2 k(k+1)}\right)\right)\right\}
$$

Fix pairwise disjoint balls $B_{1}, \ldots, B_{l}$ in $\Omega$ such that $\sum_{i=1}^{l} \mathcal{L}_{n}\left(B_{i}\right)<\delta$. From (3.3) we obtain $\operatorname{diam}\left(B_{i}\right)<\frac{1}{k}-\frac{1}{k+1}(i \in\{1, \ldots, l\})$. Thus (3.2) gives that 
either $B_{i} \subset \Omega_{k+1}$ or $B_{i} \subset \Omega \backslash \Omega_{k}$ for every $i$. Hence we obtain from the definition of $\delta_{1}$ and $k$ that

$$
\begin{aligned}
\sum_{i} \operatorname{osc}_{\lambda B_{i}}^{n} f & \leq \sum_{i: B_{i} \subset \Omega_{k+1}} \operatorname{osc}_{\lambda B_{i}}^{n} f+\sum_{i: B_{i} \subset \Omega \backslash \Omega_{k}} \operatorname{osc}_{\lambda B_{i}}^{n} f \\
& \leq \sum_{i: B_{i} \subset \Omega_{k+1}} \operatorname{osc}_{\lambda B_{i}}^{n} f+V_{\lambda}^{n}\left(\Omega \backslash \Omega_{k}\right) \\
& \leq \varepsilon+\varepsilon \\
& =2 \varepsilon
\end{aligned}
$$

and the proof is finished

The following theorem [3: Theorem 3.1] gives us the opportunity to use any $\lambda \in(0,1)$ in the definition of the classes $A C_{\lambda}^{n}$ and $B V_{\lambda}^{n}$. We will use this fact in the proof of Theorem 3.6.

Theorem 3.5. Let $0<\lambda_{1}<\lambda_{2}<1$ and $f: \Omega \rightarrow \mathbb{R}^{m}$. Then $B V_{\lambda_{1}}^{n}(\Omega)=$ $B V_{\lambda_{2}}^{n}(\Omega)$ and $A C_{\lambda_{1}}^{n}(\Omega)=A C_{\lambda_{2}}^{n}(\Omega)$.

Now we can prove the main result of this section.

Theorem 3.6. Let $n \geq 2$ and $0<\lambda<1$. Suppose that the mapping $F: \Omega \rightarrow \mathbb{R}^{n}$ is $K$-quasiconformal and $f: \Omega \rightarrow \mathbb{R}$. Then:

(i) $f \circ F^{-1} \in B V_{\lambda}^{n}(F(\Omega)) \quad \Longrightarrow \quad f \in B V_{\lambda}^{n}(\Omega)$

(ii) $f \circ F^{-1} \in A C_{\lambda}^{n}(F(\Omega)) \Longrightarrow f \in A C_{\lambda}^{n}(\Omega)$.

Proof. Let us first suppose that $f \circ F^{-1} \in B V_{\lambda}^{n}(F(\Omega))$. Thanks to Theorem 3.5 we can suppose that $\lambda=\frac{1}{2}$. We will prove that $f \in B V_{\frac{\rho 0}{2}}^{n}(\Omega)$. Recall that the constant $0<\rho_{0}<1$ comes from (3.1).

Suppose that $B_{i}=B\left(x_{i}, r_{i}\right) \subset \Omega$ are pairwise disjoint balls. Clearly,

$$
F\left(B\left(x_{i}, \frac{\rho_{0}}{2} r_{i}\right)\right) \subset B\left(F\left(x_{i}\right), \operatorname{osc}_{B\left(x_{i}, \frac{\rho_{0}}{2} r_{i}\right)} F\right) .
$$

Thanks to (3.1), for $r=\frac{\rho_{0}}{2} r_{i}$ and $x=x_{i}$ we have

$$
\begin{aligned}
B( & \left.F\left(x_{i}\right), \operatorname{2osc}_{B\left(x_{i}, \frac{\rho_{0}}{2} r_{i}\right)} F\right) \\
& \subset B\left(F\left(x_{i}\right), 4 \sup _{\left\{a:\left|x_{i}-a\right| \leq \frac{\rho_{0}}{2} r_{i}\right\}}\left|F\left(x_{i}\right)-F(a)\right|\right) \\
& \subset B\left(F\left(x_{i}\right), \inf _{\left\{b:\left|x_{i}-b\right|=\frac{1}{\rho_{0}} \frac{\rho_{0}}{2} r_{i}\right\}}\left|F\left(x_{i}\right)-F(b)\right|\right) \\
& \subset F\left(B\left(x_{i}, \frac{1}{2} r_{i}\right)\right) .
\end{aligned}
$$


Hence the balls $\widetilde{B}_{i}=B\left(F\left(x_{i}\right), 2 \operatorname{osc}_{B\left(x_{i}, \frac{\rho_{0}}{2} r_{i}\right)} F\right)$ are pairwise disjoint in $F(\Omega)$. Thus (3.4) gives us

$$
\begin{aligned}
\sum_{i} \operatorname{osc}_{B\left(x_{i}, \frac{\rho_{0}}{2} r_{i}\right)}^{n} f & =\sum_{i} \operatorname{osc}_{F\left(B\left(x_{i}, \frac{\rho_{0}}{2} r_{i}\right)\right)}^{n} f \circ F^{-1} \\
& \leq \sum_{i} \operatorname{osc}_{B\left(F\left(x_{i}\right), \operatorname{osc}_{B\left(x_{i}, \frac{\rho_{0}}{2} r_{i}\right)}^{n} F\right)} f \circ F^{-1} \\
& =\sum_{i} \operatorname{osc}_{\frac{1}{2} \widetilde{B}_{i}}^{n} f \circ F^{-1} \\
& \leq V_{\frac{1}{2}}^{n}\left(f \circ F^{-1}, F(\Omega)\right)
\end{aligned}
$$

It follows that $V_{\frac{\rho 0}{2}}^{n}(f, \Omega) \leq V_{\frac{1}{2}}^{n}\left(f \circ F^{-1}, F(\Omega)\right)<\infty$.

Now let us suppose that $f \circ F^{-1} \in A C_{\lambda}^{n}(F(\Omega))$. As before we can assume that $\lambda=\frac{1}{2}$. From the conclusions above we obtain $f \in B V_{\frac{\rho_{0}}{2}}^{n}(\Omega)$. Thanks to Lemma 3.4 and Theorem 3.5 it is enough to prove that $f \in A C_{\frac{\rho_{0}}{2}, \text { loc }}^{n}(\Omega)$.

Fix $\varepsilon>0$ and $\Omega^{\prime} \subset \Omega$ such that $\overline{\Omega^{\prime}} \subset \Omega$. Choose $\delta_{1}$ from the definition of $A C_{\frac{1}{2}}^{n}(\Omega)$ for function $f \circ F^{-1}$. By [4: Theorem 4.3], quasiconformal mappings are locally absolutely continuous and therefore $F \in A C_{\lambda}^{n}\left(\Omega^{\prime}\right)$. Hence for a given $\varepsilon_{1}=\frac{\delta_{1}}{2^{n}}$ we can choose $\delta_{2}$ from the definition of $A C_{\frac{\rho_{0}}{2}}^{n}\left(\Omega^{\prime}\right)$ for the function $F$.

Suppose that the balls $B_{i}=B\left(x_{i}, r_{i}\right) \subset \Omega^{\prime}$ are pairwise disjoint and $\sum_{i} \mathcal{L}_{n}\left(B_{i}\right)<\delta_{2}$. As before we obtain (3.4) and (3.5). Therefore the balls

$$
\widetilde{B}_{i}=B\left(F\left(x_{i}\right), 2 \operatorname{osc}_{B\left(x_{i}, \frac{\rho_{0}}{2} r_{i}\right)} F\right)
$$

are pairwise disjoint in $F\left(\Omega^{\prime}\right)$. Further, $\sum_{i} \mathcal{L}_{n}\left(B_{i}\right)<\delta_{2}$ and the definition of $\delta_{2}$ give us

$$
\sum_{i} \mathcal{L}_{n}\left(\widetilde{B}_{i}\right)=2^{n} \sum_{i} \operatorname{osc}_{B\left(x_{i}, \frac{\rho_{0}}{2} r_{i}\right)}^{n} F \leq 2^{n} \varepsilon_{1}=2^{n} \frac{\delta_{1}}{2^{n}}=\delta_{1}
$$

Analogously to (3.6) we obtain from the definition of $\delta_{1}$ that

$$
\sum_{i} \operatorname{Osc}_{B\left(x_{i}, \frac{\rho_{0}}{2} r_{i}\right)}^{n} f \leq \sum_{i} \operatorname{Osc}_{\frac{1}{2} \widetilde{B}_{i}}^{n} f \circ F^{-1}<\varepsilon
$$

and the proof is finished

The inverse mapping to a quasiconformal mapping is also quasiconformal [7: Corollary 13.3] and hence we have the following 
Corollary 3.7. Let $0<\lambda<1, n \geq 2$ and let $f: \Omega \rightarrow \mathbb{R}^{m}$. Suppose that $F: \Omega \rightarrow \mathbb{R}^{n}$ is a quasiconformal mapping. Then:

(i) $f \in B V_{\lambda}^{n}(\Omega) \quad \Longleftrightarrow f \circ F^{-1} \in B V_{\lambda}^{n}(F(\Omega))$

(ii) $f \in A C_{\lambda}^{n}(\Omega) \Longleftrightarrow f \circ F^{-1} \in A C_{\lambda}^{n}(F(\Omega))$.

The following elementary example shows that the assumption $f \in B V_{\lambda}^{n}$ from the definition of the class $A C_{\lambda}^{n}$ is important in Theorem 3.6.

Example 3.8. Let $0<\lambda<1$. There exists a domain $\Omega \subset \mathbb{R}^{2}$ and a 1-quasiconformal mapping $F: \Omega \rightarrow \mathbb{R}^{2}$ such that $f \circ F^{-1}$ is $2, \lambda$-absolutely continuous on $F(\Omega)$ but $f$ is not $2, \lambda$-absolutely continuous on $\Omega$.

Indeed, set $\Omega=\{[x, y]: x>0\}$ and $F(x, y)=\left[\frac{x}{x^{2}+y^{2}}, \frac{-y}{x^{2}+y^{2}}\right]$. In other words, for $z \in \mathbb{C}$ we define $F(z)=\frac{1}{z}$ (thus also $F^{-1}(z)=\frac{1}{z}$ ). It is well known that the mapping $\frac{1}{z}$ is conformal and hence also 1-quasiconformal [7: Theorem 8.1]. Plainly, $F(\Omega)=\{[x, y]: x>0\}$. Put

$$
\tilde{f}(x)=\sum_{k=1}^{\infty} \max \{0,1-\operatorname{dist}(x,[2 k, 0])\} .
$$

Clearly, $\tilde{f}$ is a Lipschitz function with Lipschitz constant 1 on $F(\Omega)$ and hence also $2, \lambda$-absolutely continuous.

Set $f=\tilde{f} \circ F$ (hence $\tilde{f}=f \circ F^{-1}$ ) and $B_{k}=B([2 k, 0], 1)$. Properties of inversion and easy computation gives us

$$
\widetilde{B}_{k}:=F^{-1}\left(B_{k}\right)=B\left(\left[\frac{\frac{1}{2 k+1}+\frac{1}{2 k-1}}{2}, 0\right], \frac{\frac{1}{2 k-1}-\frac{1}{2 k+1}}{2}\right)
$$

for every $k \in \mathbb{N}$. From $\operatorname{osc}_{\widetilde{B}_{k}} f \geq 1$ and $\operatorname{diam} \widetilde{B}_{k} \rightarrow 0$ we obtain that $f$ is not $2, \lambda$-absolutely continuous.

It is not difficult to prove that the condition $\mathcal{L}_{n}(\Omega)<\infty$ guarantees that any $n, \lambda$-absolutely continuous function $f$ on $\Omega$ satisfies $f \in B V_{\lambda}^{n}(\Omega)$. Hence such an example can exist only if $\mathcal{L}_{n}(F(\Omega))=\infty$ in view of Theorem 3.6.

\section{Continuous homeomorphisms $F: B V_{\lambda}^{n} \rightarrow B V_{\lambda}^{n}$}

In this section we will use ideas of Gold'stein, Gurov and Romanov [2]. They proved that a homeomorphism $F: \Omega \rightarrow \mathbb{R}^{n}$ which induces a bounded operator from $W^{1, n}(F(\Omega))$ to $W^{1, n}(\Omega)$ is a quasiconformal mapping (see [2] for details and $[3]$ for the history of similar problems).

Let us denote $F_{v}^{\prime}(x)=\lim _{r \rightarrow 0} \frac{|F(B(x, r))|}{|B(x, r)|}$. We shall need the following connection between $F_{v}^{\prime}$ and the Jacobian of $F$ [7: Theorems 24.2 and 24.4]. 
Theorem 4.1. Let $F: \Omega \rightarrow \mathbb{R}^{n}$ be a homeomorphism. Then:

(i) $F_{v}^{\prime}<\infty$ almost everywhere.

(ii) $F_{v}^{\prime}$ is a measurable function.

(iii) For each measurable set $A \subset \Omega,|F(A)| \geq \int_{A} F_{v}^{\prime}(x) d x$.

(iv) If $F$ is differentiable at $x$ and $J_{F}(x)$ is the Jacobi matrix of $F$ at $x$, then $F_{v}^{\prime}=\left|J_{F}(x)\right|$.

Lemma 4.2. If a homeomorphism $F: \Omega \rightarrow \mathbb{R}^{n}$ induces the bounded operator $F^{\star}: B V_{\lambda}^{n}(F(\Omega)) \rightarrow B V_{\lambda}^{n}(\Omega)$, then $F$ is differentiable almost everywhere on $\Omega$.

Proof. Fix $R>0$. The mapping $F$ is a homeomorphism and therefore the set

$$
A_{R}=\{x \in \Omega: F(x) \in B(0, R)\}=F^{-1}(B(0, R))
$$

is open. Fix $1 \leq i \leq n$. Plainly, there is a Lipschitz function $f: F(\Omega) \rightarrow \mathbb{R}$ such that

$$
f(x)= \begin{cases}x_{i} & \text { for } x \in F(\Omega),|x|<R \\ 0 & \text { for } x \in F(\Omega),|x|>R+1 .\end{cases}
$$

Hence $f \in B V_{\lambda}^{n}(F(\Omega))$ implies $F^{\star} f=f \circ F \in B V_{\lambda}^{n}(\Omega)$. If $|F(x)|<R$, then $f \circ F=F_{i}(x)$. Thus $F_{i}(x) \in B V_{\lambda}^{n}\left(A_{R}\right)$. Functions from $B V_{\lambda}^{n}(A)$ are differentiable almost everywhere on $A$ for every open set $A$ (see [6: Theorem 3.3] and [4: Theorem 3.4] for details) and hence $F_{i}$ is differentiable almost everywhere on $A_{R}$. Since $A_{R} \rightarrow \Omega$ as $R \rightarrow \infty$ we obtain that $F_{i}$ is differentiable almost everywhere on $\Omega$

In the proof of Theorem 4.4 below we will need the following elementary lemma [2: Lemma 3.5]:

Lemma 4.3. Let $F: \Omega \rightarrow \mathbb{R}^{n}$ be a continuous mapping and $G \subset \mathbb{R}^{k}$. Suppose that $\left\{K_{y}\right\}_{y \in G}$ is a family of pairwise disjoint compact sets such that $K_{y} \subset F(\Omega)$. Then $\mathcal{L}_{n}\left(F^{-1}\left(K_{y}\right)\right)=0$ for all $y \in G$ except possibly a countable subset of $G$.

Theorem 4.4. Let $0<\lambda \leq 1$ and $n \geq 2$. If a homeomorphism $F$ : $\Omega \rightarrow \mathbb{R}^{n}$ induces the bounded operator $F^{\star}: B V_{\lambda}^{n}(F(\Omega)) \rightarrow B V_{\lambda}^{n}(\Omega)$, then $F \in W_{\mathrm{loc}}^{1, n}(\Omega)$ and there is a number $K$ such that

$$
\left|\nabla F_{i}\right|^{n} \leq K F_{v}^{\prime}(x)
$$

for almost all $x \in \Omega$ and for all $i=1,2, \ldots, n$.

Proof. In this proof we will follow the ideas from [2: Theorem 3.6]. By Theorem 4.1, $F_{v}^{\prime}(x)<\infty$ a.e. Fix $\varepsilon>0$ and a point $x_{0} \in \Omega$ such that 
$F_{v}^{\prime}\left(x_{0}\right)<\infty$. There is $r_{0}$ such that for all $r \in\left(0, r_{0}\right)$ we have

$$
\begin{aligned}
\left|F\left(B\left(x_{0}, 2 r\right)\right)\right| & \leq\left(F_{v}^{\prime}\left(x_{0}\right)+\varepsilon\right)\left|B\left(x_{0}, 2 r\right)\right| \\
& =\left(F_{v}^{\prime}\left(x_{0}\right)+\varepsilon\right) 2^{n}\left|B\left(x_{0}, r\right)\right| .
\end{aligned}
$$

Set $M=\left(F_{v}^{\prime}\left(x_{0}\right)+\varepsilon\right) 2^{n}$. Let us call a cube $Q \quad h$-regular if all its edges are parallel to the coordinate axes, the length of the edge is $h$ and every vertex has the form $\left[k_{1} h, \ldots, k_{n} h\right]$ where $k_{1}, \ldots, k_{n}$ are integers. Fix $r<r_{0}$ and choose $h>0$ such that

$$
h<\frac{1}{2 \sqrt{n}} \operatorname{dist}\left(F\left(S\left(x_{0}, 2 r\right)\right), F\left(S\left(x_{0}, r\right)\right)\right) .
$$

Let $A$ be the union of all $h$-regular cubes $Q$ such that $Q \cap F\left(B\left(x_{0}, r\right)\right) \neq \emptyset$. It is evident that

$$
F\left(B\left(x_{0}, r\right)\right) \subset A \subset F\left(B\left(x_{0}, 2 r\right)\right) .
$$

Fix $j \in\{1, \ldots, n\}$ and let us focus on the $j$-th coordinate. Denote the hyperplanes $x_{j}=t h$ by $L_{t}$. The hyperplanes $L_{m}$ ( $m$ an integer) divide $\mathbb{R}^{n}$ into layers

$$
Z_{m}=\left\{x \in \mathbb{R}^{n}: m h<x_{j}<(m+1) h\right\} .
$$

Put $A_{m}=Z_{m} \cap A$.

For every $A_{m}$ we construct three functions

$$
\begin{aligned}
& \psi_{m, 1}=x_{j}-m h \\
& \psi_{m, 2}=(m+1) h-x_{j} \\
& \psi_{m, 3}=\frac{h}{2}-\operatorname{dist}\left(P_{j}(x), P_{j}\left(A_{m}\right)\right) .
\end{aligned}
$$

Here $P_{j}: \mathbb{R}^{n} \rightarrow \mathbb{R}_{j}^{n-1}$ is the orthogonal projection of $\mathbb{R}^{n}$ onto $\mathbb{R}_{j}^{n-1}$. Consider the functions

$$
\psi_{m}=\max \left\{0, \min \left\{\psi_{m, 1}, \psi_{m, 2}, \psi_{m, 3}\right\}\right\} \quad \text { and } \quad \psi=\sum_{m} \psi_{m}
$$

Put

$$
E=\{x \in G: \psi(x) \text { is not differentiable at } x\} .
$$

It follows from the definition of $\psi$ that:

(1) $\operatorname{supp}(\psi) \subset F\left(B\left(x_{0}, 2 r\right)\right)$

(2) $\psi$ is Lipschitz with constant 1

(3) $\psi \in B V_{\lambda}^{n}(F(\Omega))$

(4) $\psi$ is differentiable almost everywhere 
(5) $\psi(x)= \pm x_{j}+$ const in all components of the set $F\left(B\left(x_{0}, r\right)\right) \backslash E$.

The set $E \cap F\left(B\left(x_{0}, r\right)\right)$ belongs to a finite union of hyperplanes $L_{t_{1}}, \ldots, L_{t_{s}}$ where $2 t_{i}$ is an integer. By Lemma 4.3, for almost all small translations $\tau_{y}$ parallel to the axis $x_{j}$ we have

$$
\left|F^{-1}\left(\tau_{y}\left(\bigcup_{i=-\infty}^{\infty} L_{\frac{i}{2}}\right) \cap F\left(\overline{B\left(x_{0}, r\right)}\right)\right)\right|=0 .
$$

Thus we can assume without loss of generality that

$$
\left|F^{-1}\left(E \cap F\left(B\left(x_{0}, r\right)\right)\right)\right|=0 .
$$

Otherwise it is possible to change the $j$-th coordinate of the point $[0, \ldots, 0]$ at the beginning of the construction of $\psi$.

By the assumption of the theorem, $F^{\star} \psi=\psi \circ F \in B V_{\lambda}^{n}(\Omega)$. It follows from (5) and (4.2) that $(\psi \circ F)(x)= \pm F_{j}(x)+$ const for almost all $x \in B\left(x_{0}, r\right)$. It is easy to see from the proof of [5: Theorem 3.2] that $B V_{\lambda}^{n}(\Omega)$ is continuously embedded into $W^{1, n}(\Omega)$. These two facts give us

$$
\begin{aligned}
\int_{B\left(x_{0}, r\right)}\left|\nabla\left(F_{j}(x)\right)\right|^{n} d x & \leq \int_{\Omega}|\nabla(\psi \circ F)|^{n} d x \\
& \leq C V_{\lambda}^{n}(\psi \circ F, \Omega) \\
& =C V_{\lambda}^{n}\left(F^{\star}(\psi), \Omega\right) .
\end{aligned}
$$

Since $F^{\star}$ is continuous we have

$$
V_{\lambda}^{n}\left(F^{\star}(\psi), \Omega\right) \leq C V_{\lambda}^{n}(\psi, \Omega)
$$

The function $\psi$ is Lipschitz with constant 1 and hence

$$
\operatorname{osc}_{B(x, s)}^{n} \psi \leq(2 s)^{n}=C|B(x, s)|
$$

for each $x$ and every $s$. Thanks to (4.5), the continuity of $\psi$ and $\operatorname{supp}(\psi) \subset$ $F\left(B\left(x_{0}, 2 r\right)\right)$ we have

$$
V_{\lambda}^{n}(\psi, \Omega) \leq C\left|F\left(B\left(x_{0}, 2 r\right)\right)\right|
$$

From (4.3), (4.4) and (4.6) it follows that

$$
\int_{B\left(x_{0}, r\right)}\left|\nabla\left(F_{j}(x)\right)\right|^{n} d x \leq C\left|F\left(B\left(x_{0}, 2 r\right)\right)\right| .
$$


By (4.1),

$$
\int_{B\left(x_{0}, r\right)}\left|\nabla\left(F_{j}(x)\right)\right|^{n} d x \leq C M\left|B\left(x_{0}, r\right)\right| .
$$

Hence

$$
\limsup _{r \rightarrow 0} \frac{1}{\left|B\left(x_{0}, r\right)\right|} \int_{B\left(x_{0}, r\right)}\left|\nabla\left(F_{j}(x)\right)\right|^{n} d x \leq C M .
$$

The Lebesgue Theorem gives us $\left|\nabla F_{j}\left(x_{0}\right)\right|^{n} \leq C\left(F_{v}^{\prime}\left(x_{0}\right)+\varepsilon\right)$ for almost all $x_{0} \in \Omega$. Letting $\varepsilon \rightarrow 0$ we obtain

$$
\left|\nabla F_{j}\left(x_{0}\right)\right|^{n} \leq C F_{v}^{\prime}\left(x_{0}\right)
$$

for almost all $x_{0} \in \Omega$. For every compact set $K \subset \Omega$ we obtain from Theorem 4.1 and (4.7) that

$$
\int_{K}\left|\nabla F_{j}(x)\right|^{n} d x \leq C \int_{K} F_{v}^{\prime}(x) d x \leq C|F(K)|<\infty .
$$

Thus $F \in W_{\mathrm{loc}}^{1, n}(\Omega)$

Thanks to Lemma 4.2 and Theorem 4.1/(iv) we obtain the following

Corollary 4.5. Let $0<\lambda \leq 1$ and $\Omega \subset \mathbb{R}^{n} \quad(n \geq 2)$. Each homeomorphism $F: \Omega \rightarrow \mathbb{R}^{n}$ that induces a bounded operator from $B V_{\lambda}^{n}(F(\Omega)$ ) to $B V_{\lambda}^{n}(\Omega)$ is quasiconformal.

Acknowledgement. The author wishes to express his thanks to Jan Malý for suggesting the problem and for many stimulating conversations.

\section{References}

[1] Csörnyei, M.: Absolutely continuous functions of Rado, Reichelderfer and Malý. J. Math. Anal. Appl. 252 (2000), 147 - 166.

[2] Gold'stein, V., Gurov, L. and A. Romanov: Homeomorphisms that induce monomorphisms of Sobolev spaces. Israel J. Math. 91 (1995), 31 - 60.

[3] Gold'stein, V. and Yu. G. Reshetnyak: Quasiconformal Mappings and Sobolev Spaces. Dordrecht: Kluwer Acad. Publ.

[4] Hencl, S.: On the notions of absolute continuity for functions of several variables. Fund. Math. 173 (2002), 175 - 189.

[5] Hencl, S. and J. Malý: Absolute continuity for functions of several variables and diffeomorphisms. Central European J. Math. 4 (2003), 690 - 705.

[6] Malý, J.: Absolutely continuous functions of several variables. J. Math. Anal. Appl. 231 (1999), 492 - 508. 
[7] Väisälä, J.: Quasi-symmetric embeddings in Euclidian spaces. Trans. Amer. Math. Soc. 264 (1981), $191-204$.

[8] Väisälä, J.: Lectures on n-Dimensional Quasiconformal Mappings. Berlin New York: Springer-Verlag 1971.

Received 04.02.2003 